\title{
Quantum correlations of colloidal particles as the root cause of topological features of the microcosm of oxyhydrate systems and their properties
}

\author{
(C) Yury I. Sukharev \\ Department of Solid State Chemistry and Nanoprocesses. Chelyabinsk State University. Br. Kashirinykh St., \\ 129. Chelyabinsk, 454000.Russia.Phone:89634602775.E-mail: Yuri_Sucharev@mail.ru
}

Keywords: Lagrangian display, electroglobal, fulleroid, multipoles, oxyhydrate gel systems, colloid clusters, spontaneous pulsation flow, the diffuse electric double layer, topological continuum, dissociative-disproportionate to mechanism, the theory of Whitney, the geometry of the caustic, quantum correlation.

\begin{abstract}
A fundamentally new method for calculating the crystallographic structures of oxyhydrate gels that transform over time is proposed. Simple features of caustics and wave fronts form two infinite series and and three exceptional features (Symmetry groups are groups of regular polyhedra in three - dimensional space, and exceptional features are symmetry groups of tetrahedron, octahedron, and icosahedron.) This follows from the Coxeter-Dynkin diagrams.

The principles of the program "COXETER" for the implementation of the method of calculation of oxyhydrate crystallographic structures.

Gel electric moments of higher even orders are the higher degrees of Laplace operators: sixth, eighth, tenth and so on orders. In this case, a General ratio is obtained, where new amplitudes and phases are obtained by adding oscillations with different phases, recorded formulas:, which are characterized by the values of currents depending on the spatial periodic structures, that is, an experimental connection between the current and the concentration of the nanoclusters of the system is obtained .

From the analysis of the experimental data it follows that the main part of the time oscillations is determined by the light elements of the clusters, the quadrupoles of which give a relatively weak current surge with a small amplitude. Along with them there are elements of the third order, the fourth, fifth and possibly sixth.

The nanoclusters are formed by the rule of "magic numbers" found experimentally in the hydroxide application in complex tin, yttrium, iron and other. The rules of "magic numbers", or the theory of magic numbers is widely used to describe nano-structures quite complicated. The role of Paterns is at the heart of everything, that is, at the heart of the structure of Reality. Reality is not collected in parts from the particles of matter in the course of evolution from the past to the future, and is all at once from the past to the future for a given pattern, that is, for specific PATTERNS, as defined by quantum theory.

Irregularities and partial randomization of the structure of the core grids form gel defects, to which small mobile clusters are attracted by electrostatic or electromagnetic forces, which are then adsorbed and located on the "defects" in accordance with their dipole moments. This circumstance is determined by the values of"magic numbers".

Cluster magic structures have a layered structure. The interior region such fulleroids be, for example, medium-structured clusters and their multipoles and octupole. If at the same time multipoles of the same name, it inevitably leads to fluctuations of cluster flows.

The areas inside the fulleroid will not be filled completely; they always remain empty. This will also lead to permanent oscillations of the cluster "filling" and is related to the filling of the fluctuations of the cluster environment. Knowing the padding settings fulleroid (fluctuations) can be identified, what are its physico-chemical characteristics.

Linear ordered REALITY, or in other terminology, historical oxyhydrate sequence - is the result of experimental wave interference (table 4) sums of historical (time) epochs-waves of equations

Moreover, the Function satisfies the condition of the Wheeler-DeWitt equation, which allows to describe the birth of a 4-dimensional space-time gel oxyhydrate phase.
\end{abstract}


[1] A.K. Guts. Physics of reality. Omsk: Publishing house of KAHN. 2012. 424p. (russian)

[2] A.D. Alexandrov. On Einstein's paradox in quantum mechanics. Reports of the USSR Academy of Sciences. 1952. Vol.84. No.2. P.263-256. (russian)

[3] Yu.I. Sucharev. Electromagnetic noise entangled states of cluster colloidal systems. Butlerov Communications. 2016. Vol.45. No.1. P.1-41. DOI: 10.37952/ROI-jbc-01/16-45-1-1

[4] Yu.I. Sukharev. Wave properties gel colloids as a cause of cluster splash. Butlerov Communications. 2016. Vol.47. No.7. C.125-160. DOI: $10.37952 /$ ROI-jbc-01/16-47-7-125

[5] B.A. Markov, and Yu.I. Sukharev. Some quantum considerations on oscillation characteristics of oxyhydrate gels. Butlerov Communications. 2016. Vol.46. No.5. P.89-94. DOI: 10.37952/ROI-jbc-01/1646-5-89

[6] B.A. Markov, Yu.I. Sukharev, and I.Yu. Apalikova. Implementation mechanism of entangled states in colloidal systems. Butlerov Communications. 2016. Vol.45. No.1. P.42-55. DOI: 10.37952/ROI-jbc01/16-45-1-42

[7] Yu.I. Sucharev, I.Yu. Apalikova. Claster Electric Spectroscopy of Colloid Chemical Oxyhydrate Systems. Switzeland, USA: Trans Tech Publications. 2015. P.585.

[8] G.I. Volovin. Circuitry analogowych hardware and digital electronic devices. Moscow: Dodeka-XXI. 2005. 528p. (russian)

[9] Carrier of the transformation of the multifunctional E-270: passport 422272-270-42885515 PS.

[10] V.I. Arnold. Features of caustics and wave fronts. Moscow: Fazis. 1996. 562p. (russian)

[11] V.I. Arnold. Theory of catastrophes. Moscow: editorial URSS. 2004.128p. (russian)

[12] Yu.I. Sukharev, B.A. Markov, O.M. Krutikova, and A.L. Kuznetsov. Form and discharge mechanism of stochastic wave clusters near the carbon-graphite recording electrodes. Butlerov Communications. 2013. Vol.34. No.4. C.21-38. ROI: jbc-02/13-34-4-21

[13] Yu.I. Sukharev, B.A. Markov, I.Yu. Apalikova, and O.M. Krutikova Cluster electric aura of colloidchemical oxyhydrate systems. Butlerov Communications. 2014. Vol.37. No.1. P.102-111. ROI: jbc02/14-37-1-102

[14] Yu.I. Sucharev, B.A. Markov, and O.M. Shanina. New principles of the research into imperfect crystallographic forms of colloidal chemical clusters. Butlerov Communications. 2013. Vol.36. No.11. P.30-43. ROI: jbc-02/13-36-11-30

[15] I.P. Suzdalev. Nanotechnology: Physics and chemistry of nanoclusters and nanomaterials. $2^{\text {nd }}$ edition, ISPR. Moscow: URSS. 2008. 589p. (russian)

[16] Yu.I. Kulakov. Theory of physical structures. Moscow. 2004. 847p. (russian)

[17] Yu.I. Sukharev. Synthesis and application of specific oxyhydrate sorbens. Moscow: Energoatomizdat. 1987. 120p. (russian) 